\title{
Phenomenological Mechanism of Inspection in Traditional Chinese Medicine
}

\author{
Ye Deng1, Zhuowei Chen ${ }^{2}$ \\ ${ }^{1}$ Department of Philosophy, Sun Yat-sen University, Guangzhou, China \\ ${ }^{2}$ School of Management, Jinan University, Guangzhou, China \\ Email: specialdy@163.com,chenzhuowei2018@outlook.com
}

How to cite this paper: Deng, Y., \& Chen, Z. W. (2019). Phenomenological Mechanism of Inspection in Traditional Chinese Medicine. Chinese Studies, 8, 42-48. https://doi.org/10.4236/chnstd.2019.82004

Received: April 29, 2019

Accepted: May 27, 2019

Published: May 30, 2019

Copyright (c) 2019 by author(s) and Scientific Research Publishing Inc. This work is licensed under the Creative Commons Attribution International License (CC BY 4.0).

http://creativecommons.org/licenses/by/4.0/

\begin{abstract}
Inspection is the crucial process to gain the physical condition of a patient before diagnosis and treatment in Traditional Chinese Medicine (TCM). Unlike the completely objective description of a patient in Western Medicine, the subjective thinking of the medical practitioners is comprised of TCM inspection. What's more, Master of Chinese medical practitioner (CMP) could make a diagnosis only with inspection and without self-ratiocinate and demonstrate the process. This article revealed how the knowledge and experiences instantly appear in a CMP's mind when he/she makes an inspection with the methods and conclusions of phenomenological philosophy. When a CMP sees a patient, he/she can "see" the symptoms of a patient with the help of intentional intuition, which is synthesized of retention. On this basis, pathogenesis knowledge will be given by the way of passive association. This conclusion drawn from phenomenological philosophy offers a new road for many problems remained pendent in TCM diagnosis and treatment.
\end{abstract}

\section{Keywords}

Traditional Chinese Medicine (TCM), Inspection, Phenomenology, Intentionality, Passive Synthesize

\section{Introduction}

Inspection means to collect information about diseases through visually observing the changes of the whole body and the local appearance of the patient (Deng, 2006). Conventional textbooks and papers of TCM focus on the study of patients, such as their appearance, body shape, various physical states, various representations and so on, while there is little research on the course of thinking consciousness of the CMP during the inspection. We try to explain the informa- 
tion given in the inspection from the perspective of phenomenology philosophy and the aspect of the structure of consciousness rather than study the behavior of consciousness from the material level of the natural science. Phenomenology, a popular philosophical trend of thought in the West in the 20th century, which was founded by Husserl, the German philosopher, is a strict philosophical method based on direct intuition and essential understanding (Ni, 2000). Phenomenology founded by Husserl, as a philosophical method based on intuition and essential knowledge, adheres to the motto of "returning to things themselves", which guides us back to the objective object of intention. This article aims to discuss a CMP's course of thinking and consciousness during the inspection using some methods and conclusions of "phenomenology of occurrence" in the late stage of his academic career.

\section{An Example: Scene of a Patient Visiting a CMP}

First of all, we imagine that a patient with symptoms of spontaneous sweat, diagnosed by a CMP as a "deficiency". Spontaneous sweat, which is a common syndrome in TCM, refers to the yang-qi of the patient cannot be fixed to the muscle surface, and the body fluid escapes, which leads to sweat in the daytime, especially during the activity, and also with the symptoms of cold and fatigue (Tietao, 2006). Deficiency, which is a common diagnostic name in TCM, refers to the pathological state of weak vital qi, low defense ability, with the clinical symptom: pale face, drooping spirits, fatigue, shortness of breath, cold, spontaneous sweat and so on (Sun, 2007). The CMP finished a complete diagnosis and treatment process after prescribing the medicine, aimed to treat deficiency, when he/she diagnosed the patient as deficiency. Imaging that in the clinic of an experienced CMP, a patient stepped into the clinic, sweating his head and neck, wearing more clothes than ordinary people and instinctively avoiding the direct blow of an air conditioner or fan. The CMP was ready to receive this new patient right after treating the last patient, and kept an eye on this patient: The phenomenon that the phenomenon: the droplets of water were flowing around the head and neck of the patient who stood in front of the doctor was "seen" by the CMP. It is conceivable that the droplets of water around the head and neck of the patient, which has the same status as his dress, posture, costume, etc., is one of visual materials that the external space scene gave to the CMP in the moment he/she looked up to the patient. The CMP's understanding of this phenomenon is a symptom of spontaneous sweat, not just a descriptive presentation of the patient's external image, and he/she diagnosed the patient as deficiency almost at the same time, all of which was done in the instant of "inspection". In the following part, we will explain this process from the perspective of phenomenological philosophy from two aspects: the intuition of the syndrome of "spontaneous sweat" and the passive occurrence of the pathogenesis of "deficiency". 


\section{Visual Passive Occurrences of Symptoms}

Husserl believes that, all spiritual activities have inherent intention characteristics (Vela, 2014). Intentionality is the basic word describing conscious behavior in Phenomenology. In a narrow sense, intention is similar to "aiming", while in a broad sense, it includes both "aiming" and "shooting", which means the whole behavior (Ni, 2014). As a CMP, it is the professional nature that he/she sees the droplets of water coming out of the patient's head and neck, which is an expression of intention (if he/she is a clothing designer, the intention may be in the patient's dress at the very beginning). The unique symptom: spontaneous sweat, is comprehensively grasp by the CMP with the phenomenon of beads of the patient's head and neck almost the same moment. "Spontaneous sweat" is a symptom name of TCM, which refers to the performance that the patient sweats naturally rather than because of external objective influence ( $\mathrm{Du}, 2013)$.

It is the functional embodiment of comprehensive grasp and meaning giving to transform from "head and neck beads" to "spontaneous sweat", which is neither certainty nor logical. This is because "spontaneous sweat" has the soul given by comprehensive grasp and meaning giving that makes it becomes a phenomenon of connotation, which the "head and neck beads" is a simple three-dimensional pictorial material. Due to the comprehensive reasons of a variety of objective factors, the CMP will not regard the beads of the patient's head and neck as the sweat because of exercise, hot weather, or even bath and getting wet in the rain. Specifically, the cognition of "spontaneous sweat" is gained from the CMP's comprehensive grasp of "bead of head and neck" of the patient, given which the meaning of "sweating", which according to the external environment (climate, no shower room nearby), local environment (population distribution within the clinic for an hour) and so on. All of these, given directly in advance before all logical functions, belong to the empirical world in which we live and provide the basis for all cognitive roles (Husserl, 2009). "A single object is highlighted from a pre-given scene and stimulates us from the world around us". The CMP's consciousness is influenced by the structured scene and given a wealth of information at the moment he/she take a glimpse to the patient before shooting intentionality to the sweat of head and neck. These messages are the comprehensive effects exerted by the inner time consciousness, which are not the same as our subjective stream of consciousness that accumulates with time. The past information is not gone, but as a "retention" that stays in the present and appears with the intuitive material of the moment. The specific mechanism is discussed in detail in another work of Husserl, Consciousness On the Phenomenology of the consciousness of Internal time (Husserl, 2017a). Even though the CMP can explain it after the event and make a rational analysis of the process of "seeing" the patient's spontaneous sweat, the instant achievement of consciousness, which does not go through this process, is only an instant active and comprehensive grasp.

Husserl divides the occurrence of consciousness into active composition (ac- 
tive occurrence) and passive occurrence ( $\mathrm{Du}, 2013)$. In intuition, meaning giving (or comprehensive grasp) reflects the initiative of the subject-"the object is the product of the objectified self-achievement", and "objectification is always a kind of self-initiative" (Husserl, 2009). However, the initiative embodied by meaning giving is actually placed under passive intuition at the productive level of active occurrence. Once the productive behaviors are gone, their products are precipitated in consciousness and become the intuitive material for passive giving. In addition, the meaning giving, which cannot be arbitrarily applied to the material, should grasp comprehensively and give the meaning according to the nature of the material, although it is initiative at first, and reflects the freedom of intentional activity. In Husserl's phenomenology, "look" and "give" are the same (Ricoeur, 1996). Intention activities constitute the direction to the object as well as the meaning. You cannot "see" an object without meaning.

How does the pathogenesis of "asthenia syndrome" appear when the CMP receives the patient's spontaneous sweat information from inspection? Association, which is the Husserl's basic law in the field of passive occurrence, can explain it.

\section{Passive Association of Pathogenesis}

Phenomenological association belongs to passive association rather than a psychologically active association in common sense. Husserl reveals the inherent occurrence form of passive association with the method of phenomenological restoration, while this area has always been obscured in the past. "Association" means "reawakening" in Logical Studies, based on the "one thing pointing to another" in a certain order and relation (Husserl, 2017b), the connection between two objects, which is not imposed externally and arbitrarily on them, is given to consciousness by themselves; An object, the phenomenon of which is guided by self-giving, can be shown phenomenologically. Passive association is a kind of pure inner relation, which has no traditional metaphysical causal relationship; it belongs to the pure realm after restoration, which completely gets rid of the premise of objective existence (Husserl, 2017b).

For example, the image of a friend of mine comes to my mind instantly who is similar to the person that I encounter when I hang out in the street. What brought my friend's image to my consciousness in that moment while he/she did not stand in front of me in the present reality and I did not deliberately recall his/her image? This is where passive association works said by Husserl (2009). Association plays two roles at this time: the awakening and the awakened, the initiating basis of which is similarity. The current consciousness of the passer-by awakens the image of one of my friend who has been precipitated in consciousness since the face of the former is similar to the latter. The image of my friend, which is the awakened, suddenly comes out as the result of association. The association embodies a kind of original passivity at this time, because the self is receptive to the result of the passive association that the image of friend can 
come into consciousness while the active self does not participate in this process, which illustrates the acceptability of consciousness. What has been precipitated in consciousness awaked by association, which will be added to the construction of the meaning of the reality consciousness, takes part in the meaning giving of the current perception. As an illustration, passer-by will be perceived as "a person like one of my friends", all my impression of whom as "something expected

"like appearance, behavior, personality, will also be involved in the further image construction of the passer-by.

What's interesting is that if I was hanging out with another person who also knew my friend, I said to him, "This guy is very similar to one of our friends" when we encountered a passer-by while he/she disagree with that. The reason is that the comprehensive grasp of the same person/thing of different people is varied. There is no common basic impression, between the passer-by who stand in front of him/her and one of his/her friend in the consciousness of another person, or that impression of the "call-up force" is weak. Our understanding of people/things is vary from person to person, because it is the passive synthesis of consciousness, the origin of which can even be traced back to the infancy, and it is not as strict as the computer recording of various dimensional parameters. "There are characteristic of tendency and a level of intensity in this wake-up, so that it can be strengthened or weakened like a force" noted by Husserl in Analyses Concerning Passive and Active Synthesis (Husserl, 2009).

Go back to the topic of TCM diagnosis and treatment. The CMP aims to treat on the basis of differentiating the pathogenesis of the patient. Patients with "deficiency" syndrome should be given Chinese medicine to replenish the deficiency, for example. The spontaneous sweat patient with sweat around his/her head and neck as an example that was discussed before was diagnosed as "deficiency" by a CMP, whose knowledge of deficiency syndrome probably gain from a series of process, from book learning, clinical follow-up, self-practice to reflection summary. Passive association starts to work at the moment the CMP' intention is projected to the patient. The first information that can be diagnosed visually by the CMP includes, but is not limited to, expression, spontaneous sweat (beads of sweat around the head and neck), cold (wear more clothes than others), etc., which are not told by the patient, as soon as the CMP looks at the patient. These symptoms of the patient may have triggered the character scene contained in the "deficiency syndrome" of the CMP's memory knowledge. At present, the CMP has only a visual perception of the patient temporarily, which is not only the general consciousness of the patient, but also a prominent way to realize the patient as a deficiency type. As a trigger, the patient's information triggers the essential concept of deficiency syndrome in the mind of CMP. The meaning of external image displayed by the patient is not given as a simple sign and image, but is given by the CMP, in whose consciousness has the memory of the "deficiency syndrome patient" in the meaning of TCM. As in the previous example, the familiar passer-by triggered the image of my friend in my consciousness; I pas- 
sively received the face of my friend that the association had given me when I looked at the passer-by. At this time, passive association-the appearance of my friend's image-can be triggered by one side of the mouth and nostrils of the passer-by with no need to see the whole body. Similarly, the overall grasp of deficiency syndrome is bound to be comprehensive, while the local symptom of the patient's spontaneous sweat can also completely trigger the passive giving of association. (To be sure, it can be further reasoned to prove whether this patient is deficiency syndrome by his/her other main physical complains: headache, fever, etc. To be sure, the patient's next complaint: headache, fever, bad wind, etc., can be further reasoned to prove whether the CMP is false. However, the passive thinking - the comprehensive grasp of deficiency syndrome - that we are concerning about has done during the inspection.

\section{Conclusion}

Taking a spontaneous sweat patient with deficiency as an example, this paper makes a phenomenological reduction of the thinking process of the moment of inspection in TCM, and the pure phenomenological deduction is carried out by descriptive means (Deng, 2018). Giving the above explanation, we find that the inspection of TCM combines the subjective thinking and previous experience of the CMP, which is totally different from the completely objective description of the patient in western medicine. The doctor has intentional intuition, which means that the abilities of integrating existing information with time consciousness in doctors, as well as the comprehensive giving of past experience under passive association, are reflected together in the instant of inspection. This exactly explains why TCM is more like an empirical medicine, with special emphasis on the importance of "following a teacher". The method and conclusion in this paper can also provide a new way of thinking about some pending issues in TCM from the perspective of phenomenological philosophy.

\section{Funding}

Project supported by the Natural Science Foundation of Guangdong Province, China (Grant No. 2018A030310100).

\section{Conflicts of Interest}

The authors declare no conflicts of interest regarding the publication of this paper.

\section{References}

Deng, T. T. (2006). Diagnosis in Chinese Medicine. Shanghai: Shanghai Scientific and Technical Publishers.

Deng, Y. (2018). An Overview Study of Traditional Chinese Medicine Phenomenology. Medicine \& Philosophy, 39, 31.

Du, Z. T. (2013). Logos, Phenomenon and Self-Knowledge. Beijing: China University of 
Political Science and Law Press.

Husserl, E. (2017a). On the Phenomenology of the Consciousness of Internal Time. L. K. $\mathrm{Ni}$, Trans. Beijing: The Commercial Press. (Original work published 1928)

Husserl, E. (2017b). The Shorter Logical Investigations II. L. K. Ni, Trans. Beijing: The Commercial Press. (Original work published 1901)

Husserl, E. (2009). Experience and Judgment. X. M. Deng \& T. G. Zhang, Trans. Beijing: Joint Publishing Co. (Original work published 1939)

Ni, L. K. (2000). The Basic Meaning of Phenomenological Movement. Social Sciences in China, No. 4, 72.

Ni, L. K. (2014). Phenomenology and Its Effects. Beijing: Commercial Press.

Paul, R. (1996). A Key to Husserl's Ideas I trans. Mil-waukee, Marquette: Bond Harris \& Acqueline Bouchard Spurlock, Trans. Milwaukee, WI: Marquette University Press.

Sun, G. R. (2007). Basic Theory of Traditional Chinese Medicine. Beijing: Traditional Chinese Medicine publishing Co.

Vela, V. (2014). Husserl. F. B. Yang, Trans. Beijing: Zhonghua Book Company. (Original work published 2000) 